\title{
Idiomaticidad e idiomatización en traducción literaria
}

\section{Idiomaticity and Idiomatization in Literary Translation}

\author{
M. ${ }^{a}$ Isabel GonZÁLEZ-REY \\ Universidad de Santiago de Compostela \\ misabel.gonzalez.rey@usc.es
}

Resumen: Traducir la idiomaticidad de un texto en otra lengua requiere del traductor un procedimiento fraseotraductológico basado en la idiomatización. Idiomatizar el texto meta (texto B) no consiste solamente en fraseologizarlo, es decir, respetar en la lengua de destino el sistema fraseológico de la lengua de origen (texto A), sino también en tener en cuenta el resto de las combinaciones que componen el texto, tanto libres como creadas por el autor. En este estudio, abordaremos pues la idiomaticidad de un texto literario, el género más resistente, en el proceso traductivo, a la fraseologización pero no necesariamente a la idiomatización. Para ello, partiremos de un estudio idiomatológico del texto A, distinguiendo, desde las máximas de desviación y elección, tres principios de combinabilidad (libre, lexicalizada y creativa) que dan lugar a cuatro tipos de construcciones (co-creaciones, unidades fraseológicas, contra-creaciones y creaciones afines) que soportarán, en el proceso traductivo, un tratamiento que garantice la misma densidad idiomática en el texto B. Lo haremos dentro de una rama de la fraseología aplicada, la fraseotraductología, entendida como aquella que se ocupa de la traducción de la idiomaticidad de un texto. Para ello, ofreceremos las pautas de un modelo fraseotraductológico que se aplicará a un pasaje del libro El Alquimista de Pablo Coelho en el par de lenguas portugués-español.

Palabras clave: Traducción literaria; fraseología; idiomaticidad; fraseologización; idiomatización; idiomatología.

M. ${ }^{a}$ Isabel GONZÁLEZ-REY Idiomaticidad e idiomatización en traducción literaria 


\begin{abstract}
Translating the idiomaticity of a text into another language requires the translator to use a phraseotranslatological procedure based on idiomatization. The process of idiomatising the target text (text B) does not only consist of phraseologising it, i.e. respecting the phraseological system of the source language in the target language (text A), but also taking into account all the other combinations that make up the text, both free and created by the author. In this study, we will therefore deal with the linguistic nature of a literary text, the genre that is most resistant, in the translation process, to phraseologisation but not necessarily to idiomatization. To do so, we will start with an idiomatological study of text $A$, distinguishing, from the maxims of deviation and choice, three principles of combinability (free, lexicalised and creative ones) that give rise to four types of constructions (co-creations, phraseological units, counter-creations and affine creations) that will support, in the translation process, a treatment that guarantees the same linguistic density in text B. We will do this within a branch of applied phraseology, phraseotranslatology, understood as that which deals with the translation of the idiomaticity of a text. To this end, we will offer the guidelines of a phraseotranslatological model that will be applied to a passage of the book The Alchemist by Pablo Coelho in the Portuguese - Spanish language pair.
\end{abstract}

Keywords: Literary translation; Phraseology; Idiomaticity; Phraseologization; Idiomatization; Idiomatology.

\title{
1. INTRODUCCIÓN
}

Traducir la idiomaticidad de un texto literario desde la perspectiva fraseológica plantea una problemática particular que se lleva abordando desde distintas perspectivas, fuera y dentro de la fraseología. En general, esa problemática se limita al modo de traducir las unidades fraseológicas (UF) de un texto fuente a un texto meta, focalizando toda la atención en esa parte fija de la lengua y relegando la parte libre de la misma, como si su traducción se diese por sentada y no planteara ningún tipo de problemas particulares o como si ambas partes no se condicionaran la una a la otra. En muchos casos, los corpus paralelos literarios ${ }^{1}$, en los que coaparecen los textos originales con sus versiones en otras lenguas, dan cuenta de las distintas soluciones de traducción que puede adoptar un traductor en función de múltiples factores (co-textuales,

1. La importancia de la lingüística de corpus en la búsqueda de la equivalencia en traducción ya fue resaltada por G. Corpas Pastor (2008, p. 66): «El uso de corpus de textos originales, equiparables en cuanto a su diseño y composición, en varias lenguas permitía observar y describir el uso real de dichas lenguas en el discurso, y, a partir de ahí, determinar la unidad de traducción y revisar la noción de equivalencia. En este contexto, los corpus paralelos se conciben como repositorios de decisiones tomadas en el proceso de la traducción y, por ende, como reflejo del conocimiento experto del traductor profesional».

M. ${ }^{a}$ Isabel GONZÁLEZ-REY Idiomaticidad e idiomatización en traducción literaria
CLINA

vol. 6-2, December 2020, 33-50

elSSN: 2444-1961

Ediciones Universidad de Salamanca - CC BY-NC-ND 
contextuales, culturales o estilísticos) para el conjunto del texto, sin hacer distingos entre la parte fraseológica y la parte libre ${ }^{2}$. Sin embargo, muchos otros reconocen los escollos que presenta la traducción de esos fraseologismos, no solo en los textos técnicos sino, y sobre todo, en los textos literarios.

Ahora bien, aun teniendo en cuenta la importancia que tiene la traducción de la fraseología en los estudios de traducción ${ }^{3}$, se echa en falta disponer de un campo de trabajo propio desde la fraseología aplicada donde se determinen unas pautas concretas que permitan abordar el proceso traductivo en su integridad. Ese campo propio, que denominamos fraseotraductología ${ }^{4}$, sirve de ámbito de estudio teórico y práctico cuyo fin es la traducción de la idiomaticidad de un texto a partir de los principios propios de la fraseología. Las soluciones de traducción que se puedan aportar desde ese ámbito y desde la aplicación de esos principios a la traducción de tipo literario permitirán arrojar luz sobre una problemática que no deja de suscitar interés para los traductores.

Por ello, abordaremos en el presente estudio estas posibles soluciones desde la idiomatología, un campo prácticamente desaparecido hoy en día, pero que recuperamos aquí para presentar un modelo fraseotraductológico en traducción literaria, basado en un procedimiento, la idiomatización, que comprende dos dimensiones: una estrecha (la idiomatización fraseológica o fraseologización) y otra ancha (la idiomatización textual). La distinción entre estos dos niveles ${ }^{5}$ nos permitirá dividir el proceso traductivo en dos fases diferenciadas para alcanzar un único propósito: la misma idiomaticidad en el texto traducido en lengua meta (texto B) que la que tiene el texto producido en lengua fuente (texto A). Ambas nociones, idiomaticidad e idiomatización, entendida la primera como resultado que alcanzar y la segunda como proceso, comparten el prefijo idio- ${ }^{6}$, también presente en el término idiotismo. La doble acepción de esta palabra a lo largo de su historia va a condicionar nuestro modelo fraseotraductológico que ilustraremos a través de un breve pasaje de la obra El Alquimista, de Pablo Coelho.

2. Es el caso de traductores como J. R. Ladmiral $(2018$, p. 9) para quien los fraseologismos son solo «palabras más largas» que las demás y que no deben recibir ningún tratamiento especial en el proceso traductivo.

3. La relevancia de la traducción de la fraseología ha sido puesta de manifiesto por primera vez en el congreso Terminología, Fraseología y Traducción, celebrado en Ginebra en 1992. Es objeto actualmente de una colección, Encuentros Mediterráneos, derivada del primer coloquio organizado en España en 2006 en torno a esta cuestión y que dirigen P. Mogorrón, de la Universidad de Alicante, y S. Mejri, de la Universidad de Paris 13.

4. Para más detalle sobre ese ámbito, ver González-Rey (2020a). En ese trabajo, distinguimos entre fraseotraductología y fraseotraducción, considerando la primera una disciplina propia de la fraseología aplicada, y la segunda un enfoque compartido por esta y otras tres disciplinas fraseológicas más que utilizan lo interlingüístico con fines diferentes: la fraseología contrastiva, la fraseografía bi/multilingüe y la fraseodidáctica.

5. Cf. González-Rey (2015).

6. Del griego ídios ('privado', 'particular', 'propio').

M. ${ }^{a}$ Isabel GONZÁLEZ-REY Idiomaticidad e idiomatización en traducción literaria
CLINA

vol. 6-2, December 2020, 33-50

elSSN: 2444-1961

Ediciones Universidad de Salamanca - CC BY-NC-ND 


\section{LA DOBLE ACEPCIÓN DE IDIOTISMO}

A nivel lexicográfico, el Trésor de la Langue Française informatisé sitúa el origen de la palabra francesa idiotisme en $1558^{7}$ y el Diccionario de la Academia Francesa (DAF) la recoge desde su primera edición en 1694 en su sentido etimológico ${ }^{8}$, añadiendo, sin embargo, que se trata de un modo de hablar que tiene «algo de irregular». A partir de su segunda edición (1718), y en todas las siguientes, el DAF señala expresamente su condición de elemento "contrario a las reglas ordinarias de la Gramática", pero defendiendo el carácter particular que tiene en cada lengua ${ }^{9}$. Ahora bien, a parte de este valor intrínseco, ambos diccionarios añaden otro extrínseco en relación con su falta de equivalencia sintáctica exacta en otras lenguas.

A partir de esta teoría de la desviación con respecto a la norma general dentro de una misma lengua o en relación con otras, surge la doble acepción del idiotismo, y por extensión la de sus hipónimos (galicismo, hispanismo, anglicismo, etc.), con un valor intrínseco, como elemento diferente (irregular) dentro de una misma lengua, y con un valor extrínseco, como elemento diferente comparado con otro de igual condición en otra lengua. Ambos aspectos están estrechamente unidos, ya que lo propio solo se puede identificar comparándolo con algo diferente. Estas dos facetas serán las que pondrán en valor los comparatistas de los siglos XIX y XX, dando nacimiento a una nueva perspectiva de estudio llamada idiomatología.

\section{LA IDIOMATOLOGÍA}

La idiomatología, como lo indica su etimología ${ }^{10}$, se define como el estudio de lo propio en lo tocante a la lengua, en sus manifestaciones orales y escritas, y la cultura. Tomada tanto en un sentido colectivo (pueblo o nación), como individual (modo de expresarse de una persona), esta definición ha servido para que distintos campos de

7. En Nouvelles récréations et joyeux devis, de Bonaventure des Périers. Cf. versión de 1561 (p. 7) Recuperado el 3 de agosto de 2020 de http://www.bvh.univ-tours.fr/Epistemon/ B693836101_A489170.pdf.

8. Procedente, por medio del latín idiotismus ('expresión propia de una lengua') del griego tardío idiotismos ('lenguaje de los simples particulares').

9. En tiempos de la llustración, los gramáticos franceses aprovecharon el aspecto «irregular» del idiotismo para abrir un debate en torno a su lugar en la lengua. Frente a los que lo tachaban de error o vicio lingüístico, De Beauzée (1765, p. 497) defendió en la Encyclopédie de Diderot et d'Alembert su legitimidad, definiéndolo como una desviación de la norma atribuible al «genio» de la lengua. En su definición llega incluso a distinguir dos clases de idiotismos: los regulares y los irregulares.

10. Del griego idi凶ma en su doble acepción como 'peculiaridad de estilo', 'lenguaje propio de un autor' y 'lengua de un pueblo o nación'.

M. ${ }^{a}$ Isabel GONZÁLEZ-REY Idiomaticidad e idiomatización en traducción literaria
CLINA

vol. 6-2, December 2020, 33-50

elSSN: 2444-1961

Ediciones Universidad de Salamanca - CC BY-NC-ND 
trabajo surgieran en torno a dos tipos de objeto de estudio: los que se interesaron por el uso de la lengua hablada (lenguas nacionales y dialectales) y los que lo hicieron centrados en el de la lengua escrita (estilos literarios de ciertos escritores). La evolución que ha tenido cada campo varía según las lenguas y los países, pero todos adoptaron, al fin y al cabo, el mismo enfoque, el comparativo, desde un punto de vista sea intrínseco, con la mirada puesta en las especificidades de lo colectivo o de lo individual, sea extrínseco, con la mirada puesta en las diferencias de estas especificidades con respecto a una entidad más amplia (lengua dialectal, nacional o extranjera) ${ }^{11}$.

Así pues, el término idiomatología nace en un inicio con una visión intrínseca orientada a las manifestaciones específicas de un modo de hablar diferente con respecto a la norma particular de una lengua determinada, describiendo los distintos elementos que permiten considerar ese modo de hablar como singular. Y esta visión centrada en las propiedades de los modos de hablar alcanza no solo aspectos fonéticos, léxicos o sintácticos, sino también fraseológicos. De ahí el término de giros idiomatológicos, empleado por P. Guiraud (1980 [1962], p. 11) para llamar las locuciones francesas, relacionando así esos giros con la idiomatología para explicar el origen de los mismos, y de paso, designar el ámbito de estudio de esos elementos.

Con una visión extrínseca situada en lo diferente entre una lengua y las demás, la idiomatología cobra otra dimensión relacionada, esta vez, con lo escrito, desde una perspectiva comparada basada en manifestaciones individuales, las de los autores literarios. En ese tipo de estudios comparados surgen cuestiones relativas a la estilística y a la traducción. Así, en el ámbito literario, la idiomatología es sinónima de estilística tal y como la entiende J. Marouzeau (1941), como forma de analizar los recursos estilísticos empleados por un autor para referirse a la expresión de las especificidades del lenguaje. En el ámbito lingüístico, en relación con la estilística tal y como la concibe Bally ${ }^{12}$, y el contacto entre lenguas, el término idiomatología es sinónimo de estilística comparad ${ }^{13}$, nombre de la disciplina creada por J.-P. Vinay (1957), centrada en el aprendizaje de la traducción y en las divergencias y similitudes de las lenguas en sus distintos aspectos (sintáctico, léxico, semántico, pero también semiótico o cultural).

11. Esta variedad de sentidos y usos atribuida a la idiomatología por parte de los distintos campos de estudio tiene que ver no solo con los tiempos científicos de la época en que surge la palabra, dados a métodos eclécticos que fueron, a veces, puestos en entredicho, sino también con reminiscencias del pasado que se originaron en la ya citada discusión enciclopédica desatada en Francia en tiempos de la llustración en torno al concepto de idiotismo.

12. Bally (1909) es el máximo exponente de la corriente lingüística de la Estilística descriptiva, inspirada en el estructuralismo de Saussure y centrada en la lengua y sus usos corrientes, tanto orales como escritos.

13. Esta denominación proviene de la obra publicada por A. Malblanc (1944), inspirada en los principios de Bally sobre la estilística, la cual, a su vez, se ha convertido en una fuente de inspiración para J.-P. Vinay y J. Darbelnet (1958).

M. ${ }^{a}$ Isabel GONZÁLEZ-REY Idiomaticidad e idiomatización en traducción literaria
CLINA

vol. 6-2, December 2020, 33-50

elSSN: 2444-1961

Ediciones Universidad de Salamanca - CC BY-NC-ND 
En definitiva, la idiomatología, entendida tanto en un sentido intrínseco como extrínseco, se fundamenta en el estudio de las especificidades de los usos colectivos o individuales de las lenguas basado en el procedimiento contrastivo. Hatzfeld (1944-46, p. 72) la define como el estudio comparado de las lenguas en todas sus manifestaciones (dialectos, lengua común o literaria, expresión de una cultura o de una mentalidad), como se desprende de sus palabras:

El verdadero método de la idiomatología parece estar destinado a ser comparativo. [...] el estudio comparado de las lenguas romances [...] podría alcanzarse por medio de la idiomatología comparada. Este fecundo campo lingüístico literario incluye necesariamente la estética del lenguaje, la psicología de las naciones y la historia de las civilizaciones.

Esta doble concepción de la idiomatología aplicada a la creación literaria y su traducción nos permite presentar un modelo fraseotraductológico apoyado en el estudio idiomatológico de un texto A con el fin de llegar, mediante la idiomatización, a mantener en el texto $\mathrm{B}$ la misma carga idiomática que posee el texto creado en origen.

\section{LA IDIOMATICIDAD EN EL PROCESO CREATIVO Y TRADUCTIVO}

La idiomaticidad es una propiedad de la lengua actualizada que resulta no solo de la presencia de expresiones lexicalizadas en el discurso sino de la combinación de construcciones tanto fijas como libres presentes en su elaboración. Como afirma A. Greimas (1960, p. 42), «siendo idiomático todo lo que es propio de una lengua dada, toda lengua es idiomática como tal|» ${ }^{14}$. Efectivamente, si una lengua se define como diferente con respecto a otra, por lógica todo en ella debe considerarse idiomático. Sin embargo, frente a esta concepción ancha de la idiomaticidad, Greimas también reconoce una estrecha que limita la idiomaticidad a la fraseología de una lengua. A partir de esta doble concepción distingue una idiomaticidad intralingüística, resultante de la comparación de un estado de la lengua con su realización ideal, y una idiomaticidad interlingüística, resultante de la comparación entre distintas lenguas. Con la primera, se puede justificar la producción textual en lengua A y con la segunda su traducción en lengua B. A continuación vamos a recordar la aplicación de estas dos concepciones en el texto literario y su traducción ${ }^{15}$.

14. Texto original: "étant idiomatique tout ce qui est propre à une langue donnée, toute langue est idiomatique en tant que telle».

15. Cf. González-Rey (2014).

M. ${ }^{a}$ Isabel GONZÁLEZ-REY Idiomaticidad e idiomatización en traducción literaria
CLINA

vol. 6-2, December 2020, 33-50

elSSN: 2444-1961

Ediciones Universidad de Salamanca - CC BY-NC-ND 


\subsection{La idiomaticidad en el proceso creativo}

Partimos de la creencia de que la creación de un texto literario se fundamenta en dos máximas generales, la de elección y la de desviación, que dan lugar a tres tipos de principios que rigen la construcción textual, a saber: el principio de combinabilidad libre ${ }^{16}$, el principio de combinabilidad fija ${ }^{17}$ y el principio de creatividad ${ }^{18}$. De la mezcla de estos principios resulta la idiomaticidad del texto creado en la lengua fuente en su doble acepción: intralingüística e interlingüística. En la primera entraría todo lo relativo a lo propio y diferente de un grupo de palabras con respecto a otro dentro de la lengua $A$; en la segunda, todo lo propio y diferente de todo un texto con respecto a otro perteneciente a la lengua $B$.

Así, en la idiomaticidad intralingüística estarían las co-creaciones, derivadas de la combinabilidad libre, las unidades fraseológicas, que formaría el tejido fraseológico ${ }^{19}$ del texto, derivadas de la combinabilidad fija, las contra-creaciones y las creaciones afines, derivadas ambas de la creatividad. Estos tres últimos grupos de palabras están unidos por la máxima de desviación. En efecto, tanto las unidades fraseológicas como las contra-creaciones son construcciones desviadas con respecto a las co-creaciones, mientras que las creaciones afines lo son, además, con respecto a las unidades fraseológicas. Así, si las co-creaciones forman parte de la norma, las unidades fraseológicas, las contra-creaciones y las creaciones afines forman parte de la desviación de esa norma. Ahora bien, existe una diferencia sustancial: la desviación de las unidades fraseológicas pertenece al uso colectivo, mientras que la de las contra-creaciones y las creaciones afines pertenece al uso individual.

Aunque se suele relacionar la máxima de desviación con la de elección para definir el estilo de un escritor, -lo que nos llevaría a considerar solo las unidades fraseológicas, las contra-creaciones y las creaciones afines como las únicas que han sido elegidas por el escritor para marcar su estilo-, entendemos que hay que incluir en esa máxima de elección el grupo de las co-creaciones, puesto que ellas también han sido elegidas para componer todo el texto, y son, además, las que sirven de referencia «normativa»

16. Cf. Las co-creaciones de J.-H. Hausmann $(1979,1998)$ o el Open choice Principle, de J. Sinclair (1991). Ambas propuestas coinciden en explicar las combinaciones gramaticales y libres que utiliza un escritor en su creación literaria a partir del uso común de la lengua.

17. Cf. El Idiom Principle o Mutual Choice Principle, de Sinclair (1991). Este principio abarca el conjunto de expresiones lexicalizadas (gramaticales y agramaticales) que usa un escritor en su composición literaria.

18. Cf. Las contra-creaciones de J.-H Hausmann $(1979,1998)$ y las creaciones afines, de D. Siepmann (2008). Ambas propuestas se diferencian por el hecho de que las contra-creaciones son combinaciones estilísticas singulares, totalmente nuevas, creadas por un escritor, mientras que las creaciones afines son creadas a partir de la desautomatización de expresiones lexicalizadas preexistentes, procedentes del Mutual-Choice principle.

19. Para más detalles sobre la noción de tejido fraseológico, ver González-Rey (2020b).

M. ${ }^{a}$ Isabel GonZÁLEZ-REY Idiomaticidad e idiomatización en traducción literaria
CLINA

vol. 6-2, December 2020, 33-50

elSSN: 2444-1961

Ediciones Universidad de Salamanca - CC BY-NC-ND 
para las demás categorías. La combinación de todas ellas contribuye a la idiomaticidad del texto creado.

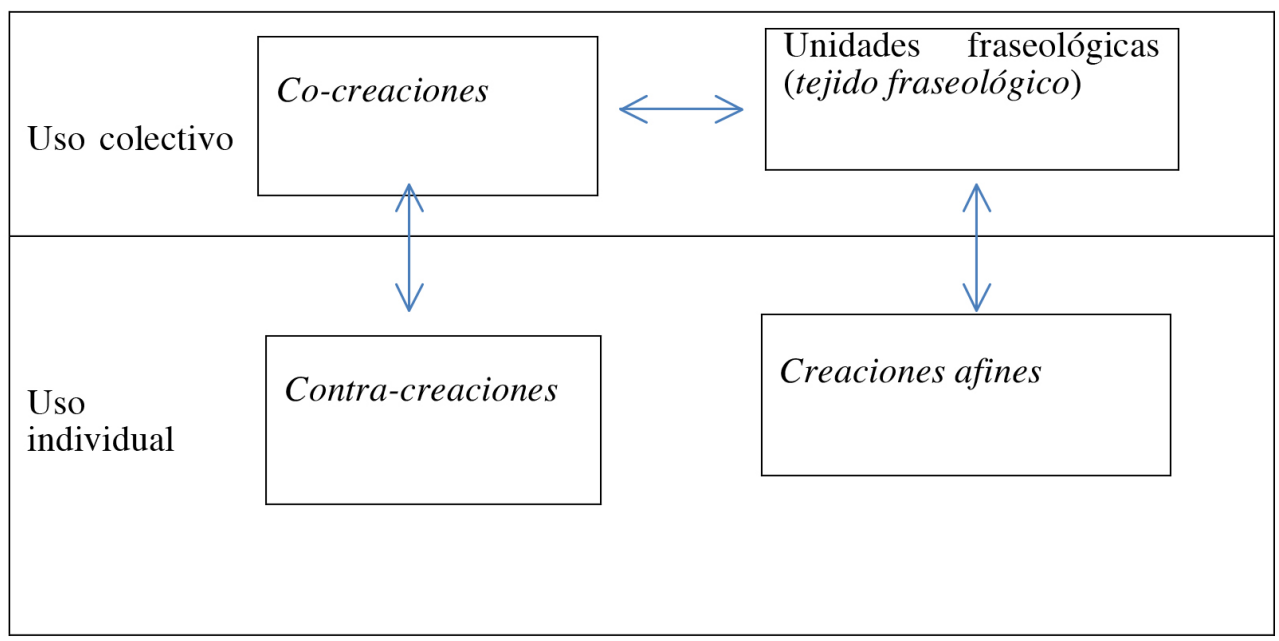

Cuadro 1. Tipos de combinaciones de uso colectivo e individual

Aunque indisociables en el momento de la escritura literaria, estos cuatro grupos se pueden distinguir unos de otros a la hora del estudio idiomatológico que un traductor haga del texto A. En dicho estudio, gracias a la máxima de desviación aplicada a cada tipo de construcción (de uso colectivo o individual), puede localizar lo derivado de cada uno de los tres principios: la escritura normativa y libre, la escritura convencional y fija, y la escritura propiamente creativa del autor. La articulación de cada parte y su fusión forman un todo heterogéneo, que responde a la máxima de elección del escritor, del cual resulta la idiomaticidad intralingüística final del texto que ilustramos en la figura siguiente:

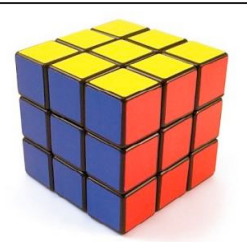

Caras separadas de los tres principios
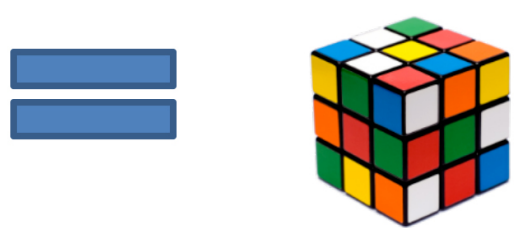

Fusión de los tres principios

Fig. 1. Idiomaticidad textual intralingüística del texto $A$

M. ${ }^{a}$ Isabel GONZÁLEZ-REY Idiomaticidad e idiomatización en traducción literaria 


\subsection{La idiomaticidad en el proceso traductivo}

Teniendo en cuenta estas máximas y estos principios en la traducción literaria, es de esperar que el texto B presente, al término del proceso traductivo, el mismo grado de idiomaticidad intralingüística que la del texto $A$, con los mismos tipos de grupos de palabras señalados anteriormente. Sin embargo, la ubicación y proporción de cada uno de ellos habrá variado en el texto meta con respecto al texto fuente, entre otros motivos porque la aplicación de las máximas de elección y desviación también habrá cambiado. Así, si la máxima de elección es previa a la de desviación en el creador del texto A y abarca los cuatro grupos por igual (mientras la de desviación alcanza solo tres de ellos), en la traducción en lengua B la máxima de desviación precede la de elección y alcanza todos los grupos.

En efecto, la idiomaticidad interlingüística se manifiesta de entrada en la desviación lingüística que presenta un texto B con respecto a un texto A. Esta desviación va a alcanzar el texto entero y condicionar todas las decisiones que tome el traductor en cada caso. Estas decisiones darán lugar a elecciones que deberán contribuir a mantener, al término del proceso traductivo, el mismo grado de idiomaticidad intrínseca en la lengua B que en la lengua A. Estas elecciones estarán sujetas a varios condicionantes:

1.- relativas al texto fuente, a saber:

a) el contenido semántico del texto A (lo que dice el texto)

b) la intencionalidad del autor (lo que quiere decir)

c) la expresividad lingüística del texto A (cómo lo dice)

2.- relativas al texto meta, a saber:

a) los recursos lingüísticos que ofrece la lengua B en su uso colectivo (con qué elementos libres y fijos puede contar el traductor)

b) la parte creativa del propio traductor (qué uso hace de la lengua de forma individual)

c) el nuevo contexto creado en lengua B (cómo encajan esos recursos unos con otros)

Dicho de otro modo, el sistema de elecciones entre autor y creador ha variado, puesto que el autor, en el momento de la creación, se ve libre de ataduras previas y decide en base al proceso creativo, mientras que el traductor asume esas decisiones y debe añadirles las suyas propias para conseguir un texto equivalente en contenido semántico e intencionalidad, pero con una expresividad lingüística diferente. Esa diferencia nace de la desviación idiomática que marca la lengua $B$ desde el inicio y va a condicionar posteriormente todas las decisiones del traductor. Por ello decimos que la máxima de desviación precede la de elección en el proceso traductivo.

Este cambio influye en la combinación de los tres principios que rigen la composición del texto literario vistos anteriormente, de tal modo que su ubicación y proporcionalidad en el texto B pueden variar con respecto al texto A. En efecto, los elementos pertenecientes a los grupos de las co-creaciones, unidades fraseológicas (tejido fraseológico), contra-creaciones y creaciones afines pueden mantener su posición en

M. ${ }^{a}$ Isabel GONZÁLEZ-REY Idiomaticidad e idiomatización en traducción literaria
CLINA

vol. 6-2, December 2020, 33-50

elSSN: 2444-1961

Ediciones Universidad de Salamanca - CC BY-NC-ND 
el texto meta o bien pasar de un grupo a otro. Así, si los recursos lingüísticos de la lengua $\mathrm{B}$ lo permiten, mantendrán su posición con un valor equivalente al del texto A; si no es así, la parte creativa del traductor buscará resolver los casos de inequivalencia mediante técnicas indirectas de traducción de tipo compensatorio. Esto incidirá necesariamente en el grado de densidad de cada uno de los grupos y modificará su proporcionalidad en el texto meta. Sin embargo, la suma de cada uno debe seguir dando el mismo valor de idiomaticidad intrínseca en ambos textos, con el fin de que la totalidad de todos ellos dé cuenta de su idiomaticidad extrínseca. llustramos la singularidad de cada texto, con una carga idiomática idéntica, en la figura siguiente:

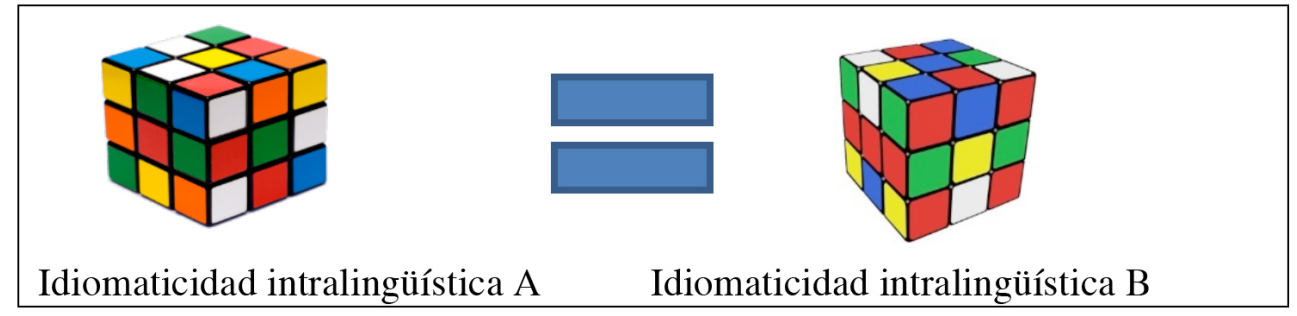

Fig. 2 ldiomaticidad interlingüística

Ahora bien, el modo de conseguir esta doble idiomaticidad, intra e interlingüística, se fundamenta en un modelo fraseotraductológico basado en el procedimiento de la idiomatización considerada en un doble sentido: uno estrecho (llamado idiomatización fraseológica o fraseologización), que abarca el grupo de las UF, y uno ancho (llamado idiomatización textual), que se ocupa del texto íntegro, es decir del resto de grupos de palabras y de su encaje entre todos ellos y el tejido fraseológico.

\section{IDIOMATIZACIÓN Y MODELO FRASEOTRADUCTOLÓGICO}

El procedimiento fraseotraductológico que proponemos en traducción literaria parte de la noción de idiomatización. Con un sufijo que la relaciona de entrada con los procedimientos, la idiomatización es un mecanismo traductivo que busca alcanzar un resultado concreto: la idiomaticidad del texto B, con un valor igual a la del texto A. Dicho esto, ese procedimiento se aplica teniendo en cuenta el doble sentido que contienen su prefijo y derivados, como hemos visto hasta aquí: un sentido estrecho, que se limita a la traducción de la fraseología del texto A al texto B; y un sentido ancho, que se ocupa de la traducción del texto en su conjunto desde la lengua $A$ hacia la lengua $B$.

M. ${ }^{a}$ Isabel GONZÁLEZ-REY Idiomaticidad e idiomatización en traducción literaria 


\subsection{La idiomatización como mecanismo traductivo}

La idiomatización, como proceder traductivo, se rige por las dos máximas vistas hasta ahora, la de desviación y la de elección. Aplicando la primera, la de desviación, lo primero que puede hacer un traductor es localizar en el texto A todos aquellos casos de equivalencia e inequivalencia con respecto a la lengua $B$, presentes en cada uno de los cuatro grupos de palabras (co-creaciones, UF o tejido fraseológico, contra-creaciones y creaciones afines). Así, podrá discernir, por un lado, todo lo que entra dentro de lo traducible mediante la aplicación de un sistema de equivalencias posibles que la propia lengua B provee y que el contexto admite, y todo lo que no.

En el primer supuesto, todas aquellas combinaciones del texto A, sean del tipo que sean, que dispongan de equivalencias en lengua B podrán ser traducidas mediante técnicas directas de traducción ${ }^{20}$. En caso de que esas combinaciones tengan más de una posibilidad de traducción, el traductor deberá decidir en función del contexto. En el segundo supuesto, en caso de inequivalencia, la traducción deberá tratarse con técnicas indirectas tales como la omisión, el préstamo, la traducción literal, la paráfrasis, la reducción o la compensación. Ahí es donde más interviene la máxima de elección, puesto que cada caso demandará una solución de traducción particular que requerirá de la pericia lingüística del traductor y de su propia capacidad creativa. La solución de traducción en lengua $B$, cuando no se ubique para cada caso en el lugar mismo que ocupe en el texto A, puede trasladarse a otro lugar del texto y cambiar así de grupo, de tal modo que cualquier elemento perteneciente al grupo, por ejemplo, de las co-creaciones puede transformarse en unidad fraseológica, contra-creación o creación afín, y así para cualquier otro elemento de cualquier otro grupo.

A partir de estos dos supuestos, proponemos un modelo fraseotraductológico que permite explicar cómo el traductor puede conseguir idiomatizar el texto B, garantizándole el mismo grado de idiomaticidad que en el texto $A$.

\subsection{Modelo fraseotraductológico}

El modelo fraseotraductológico presentado en González-Rey (2020a) consiste en distinguir dos etapas en el proceso traductológico: la idiomatización del texto $B$, en un sentido estrecho, o etapa de fraseologización (o idiomatización fraseológica), y la idiomatización del mismo, en un sentido ancho, o etapa de idiomatización textual.

La etapa de fraseologización (o idiomatización fraseológica) consiste en traducir las UF del tejido fraseológico del texto de origen mediante técnicas directas de traducción ${ }^{21}$. Para ello, se propone las siguientes pautas: 1) extraer el tejido fraseológico del

20. Cf. Las técnicas directas e indirectas de Vinay y Darbelnet (1958).

21. Aludimos aquí a la clásica división establecida por Vinay y Darbelnet (1958) entre técnicas directas y técnicas oblicuas.

M. ${ }^{a}$ Isabel GONZÁLEZ-REY Idiomaticidad e idiomatización en traducción literaria
CLINA

vol. 6-2, December 2020, 33-50

elSSN: 2444-1961

Ediciones Universidad de Salamanca - CC BY-NC-ND 
texto de origen, basándonos en la tipología de sus elementos (enunciados fraseológicos: pragmatemas y paremias; y sintagmas fraseológicos: locuciones y colocacio$n{ }^{22}$ ) y las funciones discursivas que desempeña cada tipo dentro del texto (como argumentadores, conectores o modalizadores $\left.{ }^{23}\right)$; 2) buscar, a continuación, las correspondencias lexicográficas de cada UF ${ }^{24}$; 3) transformar, después, estas correspondencias en equivalencias, ordenándolas según su grado de afinidad (total, parcial o nula) ${ }^{25}$.

Al delimitar con estas pautas el tejido fraseológico y distinguirlo de las co-creaciones dentro de los grupos de palabras de uso colectivo (principios de combinabilidad libre y fija), resulta más fácil reconocer también, dentro de los grupos de palabras de uso individual (principio de creatividad), el grupo de las creaciones afines. Por otra parte, al iniciar el proceso traductivo por esta etapa, los casos de inequivalencia permiten al traductor optar por utilizar la traducción literal o el préstamo, o bien repartir la idiomaticidad fraseológica entre los demás grupos del texto.

En cuanto a la etapa de idiomatización textual, consiste en traducir las demás combinaciones, las derivadas tanto del principio de combinabilidad libre y como las de creatividad. Dentro de las combinaciones del primer principio se incluye todo texto que no haya sido identificado durante la etapa de fraseologización como producto del principio de combinabilidad fija, y que, por lo tanto, puede entrar en la categoría de las combinaciones libres. El estudio idiomatológico llevado a cabo anteriormente nos habrá permitido detectar las que son de uso corriente y colectivo (las co-creaciones) y las que son de uso individual y creativo. Dentro de estas últimas habremos definido las que son producto, por un lado, de la deslexicalización de unidades fraseológicas (las creaciones afines) y, por otro, de la combinación impredecible y original de elementos libres de la lengua (las contra-creaciones). En el proceso de idiomatización textual el traductor tendrá que tratar las creaciones afines y las contra-creaciones mediante la adaptación a la cultura de la lengua meta para que esas creaciones singulares del autor consigan el mismo efecto en el lector del texto traducido que el producido en el del texto original. La pericia del traductor ya no se basa en una simple búsqueda de elementos lingüísticos ya disponibles en la lengua, con la ayuda de soportes externos

22. Para más detalles sobre esta tipología, ver González-Rey (2018).

23. Para más detalles sobre estas funciones discursivas, ver González-Rey (2020b).

24. Distinguimos entre correspondencias lexicográficas y equivalencias discursivas, de acuerdo con Lederer (2002). Así en esta fase, la búsqueda de correspondencias se hace a través de la traducción "estática», es decir mediante diccionarios, tanto en formato papel como electrónico, con el fin de reunir el mayor número posible de unidades de traducción para cada UF.

25. Estas pautas se pueden completar con otras dos en caso de inseguridad por parte del traductor frente a la presencia o no de una UF, a saber: 4) verificar las concordancias intralingüísticas en las que pueden aparecer estas UF en la lengua A, y comprobar su coincidencia con las concordancias interlingüísticas reunidas en la lengua B; 5) comprobar la concordancia del texto $A$ con respecto a las recogidas en esa lengua y retener entre las reunidas en lengua $B$ la que mejor se adapte al texto B.

M. ${ }^{a}$ Isabel GONZÁLEZ-REY Idiomaticidad e idiomatización en traducción literaria
CLINA

vol. 6-2, December 2020, 33-50

elSSN: 2444-1961

Ediciones Universidad de Salamanca - CC BY-NC-ND 
o tecnicismos. En estos casos, la idiomatización de las creaciones afines y las contra-creaciones dependerá de la parte creativa que posea el propio traductor, en simbiosis con la del autor, sobre todo, una vez más en los casos de inequivalencia.

La etapa de idiomatización del texto B finalizará con la conexión del tejido fraseológico con su entorno textual (enlace intraidiomático) y con la comparación de la idiomaticidad del mismo con la del texto A. Después de las etapas de idiomatización fraseológica (o fraseologización) e idiomatización textual, la proporción en la que han sido utilizados los 3 principios en el texto de origen puede haber variado durante el proceso traductivo. En efecto, puede que ni todas las UF ni las co-creaciones ni las contra-creaciones del texto A hayan mantenido su cantidad ni su ubicación en el texto B. Aun así, lo que importa es que el texto traducido resulte «idiomático» en su conjunto para su potencial lector, del mismo modo que lo es el texto original para el suyo.

\subsection{Estudio de caso}

Aplicaremos ahora esta propuesta fraseotraductológica a un breve pasaje de la obra de Paulo Coelho, O Alquimista ${ }^{26 / E l}$ Alquimista ${ }^{27}$, comparando cada frase del original en portugués (A) con su traducción en español (B), acompañada de la explicación de las técnicas de fraseologización e idiomatización textual utilizadas en cada caso.

1) A: O livro que mais interessou ao rapaz contava a historia dos alquimistas famosos.

B: El libro que más interesó al muchacho contaba la historia de los alquimistas famosos.

En la frase $A$, no se encuentra ninguna $U F^{28}$ y se constata que el texto ha sido creado según el principio de combinabilidad libre (co-creaciones). Se inicia pues el proceso traductivo directamente con la etapa de idiomatización textual. Su transvase a la frase B se hace según ese mismo principio, recurriendo a la utilización de la lengua común, tanto desde el punto de vista léxico como sintáctico. Se puede observar que la carga idiomática no ha variado de un texto a otro, ni en proporción ni en ubicación. Desde la perspectiva idiomatológica, hay equivalencia total entre las concordancias A y B.

2) A: Eram homes que tinham dedicado sua vida inteira a purificar metais nos laboratórios;

26. Versión PDF descargable en línea [www.paulocoelho.com.br] (p. 47).

27. Versión PDF descargable en línea [https://freeditorial.com/es/books/el-alquimista] (p. 46).

28. No hay que tomar la secuencia 'contar la historia de' por una UF propiamente dicha, aunque existe la colocación 'contar historias' donde la base «historias» puede ir con otro colocativo como «narrar», «relatar», pero no «decir», o bien donde el formativo «contar» podría ir con otras bases como «cuentos», «chistes», «anécdotas», etc. Cf. A. Robles-Sáez (2011, pp. 65-66). Por lo tanto, en el texto hay que tomar esa secuencia como una combinación libre.

M. ${ }^{a}$ Isabel GONZÁLEZ-REY Idiomaticidad e idiomatización en traducción literaria
CLINA

vol. 6-2, December 2020, 33-50

elSSN: 2444-1961

Ediciones Universidad de Salamanca - CC BY-NC-ND 
B: Eran hombres que habían dedicado toda su vida a purificar metales en los laboratorios;

En la frase A, se encuentra la UF 'dedicar sua vida inteira a', una colocación que desempeña una función de argumentador verbal en el texto. Se inicia, entonces, la etapa de fraseologización, extrayéndola de la frase y analizando su forma y su función; se comprueban luego sus correspondencias lexicográficas en lengua B ('consagrar/ dedicar (toda) la/su vida a [algo/hacer algo]'; 'consagrar/dedicar la/su vida entera a [algo/hacer algo]') para conseguir la que más se podría acercar a la UF del texto A. Si hay varias, se elige la que pueda tener mayor grado de equivalencia discursiva, pero antes de seleccionarla, se verifican sus concordancias en la lengua B para asegurarnos de su frecuencia de uso y su sentido. En este caso, se observa que la fraseologización se produce según el principio de combinabilidad fija por equivalencia parcial: 'dedicar sua vida inteira a' = 'dedicar toda su vida a [hacer algo]', a pesar de que hubiese sido posible hacerlo por equivalencia total. A continuación se inicia la etapa de idiomatización textual, traduciendo las demás combinaciones libres creadas por co-creación, y trasladándolas a B según el mismo procedimiento. En este caso también se puede observar que la carga idiomática no ha variado de un texto a otro, ni en proporción ni en ubicación, ya que desde la perspectiva idiomatológica hay equivalencia total entre las concordancias $\mathrm{A}$ y $\mathrm{B}$.

3) A: acreditavam que se um metal fosse cozinhado durante muitos e muitos anos, terminaria se libertando de todas as suas propiedades individuais, e em seu lugar sobrava apenas a Alma do Mundo.

B: creían que si un metal se mantenía permanentemente al fuego durante muchos años, terminaría liberándose de todas sus propiedades individuales y sólo restaría el Alma del Mundo.

En la frase A, se encuentra una UF, 'em seu lugar', de tipo adverbial, motivo por el que se debería haber iniciado la fase de fraseologización, siguiendo los mismos pasos ya mencionados en 2). Sin embargo, se advierte en B que el traductor optó por obviar su traducción y que no usó ninguna técnica compensatoria facultada de mantener la misma carga fraseológica que en A. Puede deberse a un simple olvido por su parte, ya que la lengua B tiene a su disposición la misma UF, 'en su lugar' y podría haber sido utilizada con el mismo sentido y la misma función que en A, respetando la intención del autor. Solo por ese motivo ya podríamos pensar que B no contiene, desde la perspectiva idiomatológica, la misma carga que A. Sin embargo, nos falta todavía analizar el resto del entorno textual, y entonces sí se observa que el texto A ha sido creado no solo según el principio de combinabilidad libre (co-creaciones), sino también según el de creatividad (contra-creaciones y creaciones afínes). Para poder comprobarlo, se procede a la fase de idiomatización textual localizando primero los casos de contra-creación en A, tales como la secuencia 'durante muitos e muitos anos', traducida en B por 'durante muchos años'. Se aprecia aquí que el autor hizo en A uso de una redundancia con evidente intención de estilo, que el traductor no quiso, aparentemente, reproducir. Sin embargo, la carga idiomática de A en esa secuencia no está del

M. ${ }^{a}$ Isabel GONZÁLEZ-REY Idiomaticidad e idiomatización en traducción literaria
CLINA

vol. 6-2, December 2020, 33-50

elSSN: 2444-1961

Ediciones Universidad de Salamanca - CC BY-NC-ND 
todo perdida, sino que solo ha sido desplazada, puesto que vemos que el traductor recurrió, a su vez, al mismo recurso (la redundancia) para traducir el predicado 'fosse cozinhado', situado justo antes, por 'se mantenía permanentemente al fuego', donde el adverbio 'permanentemente' resulta redundante con respecto al verbo 'mantener', que indica precisamente la «permanencia». A esta técnica, llamada de compensación, el autor añade otro recurso, la traducción por creación afín, ya que el verbo 'cozinhar' fue traducido por la secuencia 'mantener al fuego', creada a partir de la UF 'poner al fuego' donde 'mantener' sirve para indicar duración donde el portugués expresa esa idea con la estructura 'estar + participio pasado'. Con este recurso, el traductor recupera la carga fraseológica «perdida» al introducir una UF modificada en su traducción, aunque no fuese en el lugar correspondiente al texto $A$. En cuanto al resto del texto de la frase $\mathrm{A}$, organizado según el principio de combinabilidad libre, se traduce a B según el mismo principio y respetando las reglas sintácticas propias de B, las cuales explican dos casos de traducción totalmente condicionados por la gramática: 'se libertando' ha sido traducido por 'liberándose', con un uso enclítico del pronombre en B mientras su uso es proclítico en A, y 'todas as suas' por 'todas sus', con ausencia de determinante ante adjetivo posesivo en B. En resumen, se puede decir que desde la perspectiva idiomatológica, la carga idiomática del texto A está presente en B en proporción aunque no en ubicación.

En definitiva, a través de estos tres ejemplos, podemos ver que el traductor es sensible a la presencia de las UF en el texto A y en consecuencia toma decisiones que pueden ser:

1) traducir la UF original, buscando un equivalente en la lengua meta, y ubicar su traducción en $\mathrm{B}$ en el mismo lugar que en el texto A, como en 2);

2) no traducir la UF original, pero buscando compensarlo traduciendo por una UF, incluso modificada, otra unidad léxica de A ubicada en otro segmento de la frase, como en 3).

Estas decisiones influyen en la traducción del entorno textual en las que van estas UF, por lo que, otra vez, tiene que decidir:

1) en el primero de los casos:

- traducir todas las co-creaciones de A por otras tantas en B, respetando las restricciones sintácticas de la lengua meta, en proporción y ubicación, como en 1) y 2);

- traducir todas las contra-creaciones y creaciones afines de A por otras tantas en B, en proporción y ubicación, siempre que sea posible.

2) en el segundo de los casos:

- traducir algún ítem por una UF, por compensación, como en 3);

- traducir las contra-creaciones de A, si no en el mismo caso, en otro segmento de la frase, también por compensación, utilizando el mismo recurso "creativo", siempre que sea posible, como en 3);

- utilizar las creaciones afines en la traducción, aunque no estén en A, para completar alguno de los recursos anteriores, como en 3).

M. ${ }^{a}$ Isabel GONZÁLEZ-REY Idiomaticidad e idiomatización en traducción literaria
CLINA

vol. 6-2, December 2020, 33-50

elSSN: 2444-1961

Ediciones Universidad de Salamanca - CC BY-NC-ND 
A la vista de este pequeño muestrario (más amplio en nuestro estudio preliminar), podemos comprobar cómo el traductor ha estado pendiente de la carga idiomática del texto A para trasladarla en su misma proporción al texto B. Aunque aplicado probablemente de modo inconsciente, el modelo fraseotraductológico que ha seguido muestra que ha tenido en cuenta primero las UF del texto A para poder, en caso de no poder traducirlas directamente, trasladar la carga fraseológica al resto del texto y así conseguir su idiomatización. Este se ve, desde la idiomatología, como una pieza entera, en el que el todo debe contribuir a ser visto como idiosincrático. La idiomatización del texto $B$ no es solo traducir de forma separada cada uno de los grupos de palabras que lo compone, sino saber que todos ellos forman parte de las soluciones traductivas y pueden invertir su proporción y ubicación con respecto al texto original en el resultado final, cuyo fin no es otro que conseguir dar al texto meta la misma carga idiomática que la del texto original.

\section{CONCLUSIÓN}

Hemos situado este estudio sobre traducción literaria en el ámbito de la fraseotraductología, disciplina que hemos definido como aquella que está centrada en la traducción de la idiomaticidad de una lengua a otra. Para ello, hemos presentado un modelo fraseotraductológico cuyo objetivo es conseguir, por un lado, un texto B con una idiomaticidad intralingüística de igual valor a la del texto $\mathrm{A}$, y por otro dos textos con una idiomaticidad interlingüística destacable que permita dar a conocer sus diferencias, y por lo tanto apreciar la singularidad de cada uno de ellos.

Para alcanzar este fin, y gracias a un estudio idiomatológico basado primero en una dimensión intrínseca de la idiomatología (como estudio individual de cada uno de los textos) y luego extrínseca (como estudio comparado de ambos), hemos distinguido cuatro grupos de combinaciones formados el amparo de las máximas de desviación y elección y regidos por tres principios de combinabilidad: dos de uso colectivo, tales como las co-creaciones (principio de combinabilidad libre) y las unidades fraseológicas o tejido fraseológico (principio de combinabilidad fija) y dos de uso individual, tales como las contra-creaciones y las creaciones afines (ambas relacionadas con el principio de creatividad).

Aplicadas estas premisas a la traducción literaria, el modelo fraseotraductológico presentado en este trabajo se ha basado en el procedimiento de la idiomatización, noción entendida en un sentido estrecho como traducción de la fraseología del texto A (etapa de fraseologización o idiomatización fraseológica), y en un sentido ancho, como traducción del resto de los grupos de palabras (idiomatización textual). Este procedimiento remata conectando el tejido fraseológico con su entorno textual (enlace intraidiomático) y con la comparación de la idiomaticidad del mismo con la del texto $\mathrm{A}$ para garantizar que el texto $\mathrm{B}$ mantiene la misma densidad idiomática que el texto $\mathrm{A}$.

M. ${ }^{a}$ Isabel GONZÁLEZ-REY Idiomaticidad e idiomatización en traducción literaria
CLINA

vol. 6-2, December 2020, 33-50

elSSN: 2444-1961

Ediciones Universidad de Salamanca - CC BY-NC-ND 
Toda esta estructura binaria, que va desde la distinción entre fraseología e idiomaticidad, por una parte, entre idiomaticidad intralingüística e interlingüistica, por otra, hasta llegar a distinguir idiomatología intrínseca e extrínseca, por otra más, tiene su origen en el doble sentido de la palabra idiotismo. A partir de esta doble acepción, cuyo origen remonta al debate iniciado en el siglo XVIII con de Beauzée, hemos podido comprobar que sigue existiendo gracias a un modelo fraseotraductológico basado en ella. Su aplicación en el estudio de un breve pasaje de la obra El Alquimista, de Pablo Coelho, nos ha permitido comprobar que estas dos acepciones de la idiomaticidad conviven sin contradicciones en la producción literaria y su traducción.

En fin de cuentas, no resulta del todo extraño que sea así, ya que, aun reconociendo que existen en la producción del texto literario diferentes niveles de combinaciones lingüísticas (libres, fraseológicas y creativas), bien es sabido que el objetivo de cualquier traducción consiste en conseguir en todo caso un texto tan idiomático en lengua meta como lo es en lengua fuente.

\section{BIBLIOGRAFÍA}

Bally, Charles. 1921 [1909]. Traité de Stylistique française. Volume I. Heidelberg: Carl Winter's Universitätsbuchhandlung.

BeauzéE, Nicolas (de). 1765. «ldiotisme». En Encyclopédie ou dictionnaire raisonné des sciences, des arts et des métiers, Vol. VIII, ed. por Jean le Rond D’alembert y Denis Diderot. París: Briasson, David l'aîné, Le Breton, Durand, 497.

CoRPAS PAstoR, Gloria. 2008. Investigar con corpus en traducción: los retos de un nuevo paradigma. Frankfurt am Main: Peter Lang.

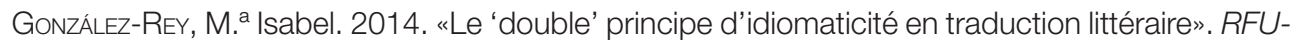
LL (Revista de Filología de la Universidad de La Laguna) 32: 227-244.

GonZÁLEz-REY, M. ${ }^{a}$ Isabel. 2015. «Fraseologización e idiomatización en traducción literaria». En Enfoques actuales para la traducción fraseológica y paremiológica: ámbitos, recursos y modalidades, ed. por Manuel Sevilla Muñoz. Madrid: Instituto Cervantes (Biblioteca Fraseológica y Paremiológica, serie «Monografías» 6, 143-160.

GonZÁLEZ-REY, M. ${ }^{a}$ Isabel. 2018. "Comment acquérir un savoir grammatical en FLE par le biais de la phraséologie». En Le même, le semblable et le différent au sein de la langue, de la littérature et de la culture dans les pays francophones, ed. por Zvonko Nikodinovski. Skopje: Faculté de philologie «Blaže Koneski» Université «Sts Cyrille et Méthode», 42-63.

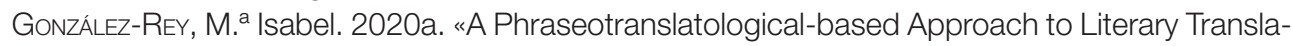
tion». En Teaching phraseology in the XXI century: new challenges, ed. por Flor Mena y Carla Stroschen. Frankfurt am Main: Peter Lang, 53-74.

GonzÁlez-Rey, M. ${ }^{a}$ Isabel. 2020b. «Didáctica del tejido fraseológico en el ámbito del Francés con Fines Universitarios (FFU)». Ciências do Léxico 9, Editora UFMS, 18-46.

GreIMAS, Algirdas. 1960. «ldiotismes, proverbes, dictons». Cahiers de Lexicologie 2: 41-61.

Guiraud, Pierre. 1980 [1962]. Les locutions françaises. París: Presses Universitaires de France (Que sais-je ?).

M. ${ }^{a}$ Isabel GONZÁLEZ-REY Idiomaticidad e idiomatización en traducción literaria
CLINA

vol. 6-2, December 2020, 33-50

elSSN: 2444-1961

Ediciones Universidad de Salamanca - CC BY-NC-ND 
HatzFeld, Helmut Anthony. 1944-46. «Nuevas investigaciones estilísticas en las literaturas románicas (1932 - 1945)». Boletín de Filología de la Universidad de Chile 4, 7-77.

Hausmann, Franz-Joseph. 1997. "Tout est idiomatique dans les langues». En La locution entre langue et usages, ed. por Michel Martins-Baltar. Fontenay/St. Cloud: ENS Éditions, 277290.

Hausmann, Franz-Joseph. 1998. «O diccionario de colocacións. Criterios de organización». En Actas do I Coloquio galego de Fraseoloxía, ed. por Xesús Ferro Ruibal. Santiago de Compostela: Xunta de Galicia, 63-81.

LADMIRAL, Jean-René. 2018. «De la phraséologie à une dialinguistique : étude programmatique». En Fraseología, Diatopía y Traducción, ed. por Pedro Mogorrón Huerta y Antonio Albaladejo-Martínez. Amsterdam/Philadelphia: John Benjamins (IVITRA Research in Linguistics and Literature, 17), 8-17.

LEDERER, Marianne. 2002. "Correspondances et équivalences faits de langue et faits de discours en traduction». En Altérité, identité, équivalence : la Traduction comme Relation, ed. por Fortunato Israël. París: Minard lettres Modernes, 17-34.

Malblanc, Alfred. 1944. Pour une stylistique comparée du français et de l'allemand. Essai de représentation linguistique comparée. París: Didier.

Marouzeau, Jules. 1941. Précis de stylistique française. París: Masson.

Robles-SÁEz, Adela. 2011. 3,000 locuciones verbales y combinaciones frecuentes. Washington D.C: Georgetown University Press.

SiEPMANN, Dick. 2008. «ldiomaticité et traduction : essai d'une systématisation». En Les séquences figées: entre langue et discours, ed. por Peter Blumenthal y Salah Mejri. Stuttgart: Steiner, 175-195.

SinclaIR, John. 1991. Corpus, Concordance, Collocation. Oxford: Oxford University Press.

VINAY, Jean-Paul. 1957. «Peut-on enseigner la traduction ? ou Naissance de la Stylistique comparée». Meta 2/4: 141-151.

VINAY, Jean-Paul \& Jean Darbelnet. 1958. Stylistique comparée du français et de l'anglais. Méthode de traduction. París: Marcel Didier.

M. ${ }^{a}$ Isabel GONZÁLEZ-REY Idiomaticidad e idiomatización en traducción literaria 